\section{CLINICAL REPORT}

\author{
Z. Patay \\ J.C. Mills \\ U. Löbel
}

A. Lambert

A. Sablauer

D.W. Ellison

\title{
Cerebral Neoplasms in L-2 Hydroxyglutaric Aciduria: 3 New Cases and Meta-Analysis of Literature Data
}

\begin{abstract}
SUMMARY: Increasing evidence suggests that patients with L2-HGA have a predisposition to cerebral neoplasms. This may be related to the pathologic accumulation of L2-HG because high amounts of 2-HG have been found in brain neoplasms that have IDH1 mutations. Our experience, on the basis of 11 previously unreported cases of L2-HGA, 3 of which developed cerebral neoplasms during the course of the disease, also supports an association between L2-HGA and cerebral neoplasms. We conducted a meta-analysis of published data, and we identified 295 patients (including our 11 patients) with L2-HGA. In 14 patients, the metabolic disorder was associated with cerebral neoplasms, suggesting an approximately $5 \%$ prevalence rate of CNS neoplasms in patients with L2-HGA; nonetheless, it may still be an underestimate. L2-HGA is an important disease "model" that provides further evidence to support the recently proposed pathogenetic role of 2-HG in the development of cerebral neoplasms.
\end{abstract}

ABBREVIATIONS: 2-HG = 2-hydroxyglutarate; IDH = isocitrate dehydrogenase; $\mathrm{L} 2-\mathrm{HG}=\mathrm{L}-2$ hydroxyglutarate; $\mathrm{L} 2-\mathrm{HGA}=\mathrm{L}$-2-hydroxyglutaric aciduria; $\mathrm{L} 2 \mathrm{HGDH}=\mathrm{L}$-2-hydroxyglutarate dehydrogenase; $\mathrm{MDH}=$ malate dehydrogenase; $\mathrm{PNET}=$ primitive neuroectodermal tumor; $\mathrm{WHO}=$ World Health Organization

$\mathbf{L}^{2}$ 2-HGA is a rare organic acidopathy. Compelling evidence suggests that the disease predisposes to the development of cerebral neoplasms. On the basis of 4 cerebral neoplasms observed in 7 patients with confirmed L2-HGA, the most comprehensive description to suggest an association between L2HGA and malignant brain tumors is that of Moroni et al. ${ }^{1}$

Our experience with patients affected by L2-HGA supports this association, which we report here alongside a meta-analysis of pertinent published data on the prevalence of brain tumors in patients with L2-HGA.

\section{Case Series and Review of Literature Data}

We have encountered 11 patients (male/female: 9:2) with L2-HGA in our clinical practice. Three of these patients developed cerebral neoplasms during the course of their disease (Fig 1). In fact, 2 of the patients were known to have L2-HGA at the time of tumor diagnosis (cases 1 and 2, presenting with a right hippocampal anaplastic astrocytoma [Fig 2] and a right frontal oligodendroglioma, respectively), whereas in the third case, the diagnosis of underlying metabolic disorder was made at the preoperative radiologic work-up of the tumor (case 3, with a left thalamic low-grade glioma). The metabolic diagnosis was subsequently confirmed by laboratory tests. The demographic, clinical and histopathologic data on these patients together with all published data on patients with L2-HGA is summarized in the Table.

We found 295 cases of L2-HGA (including our 11 patients) in the

Received June 8, 2011; accepted after revision, August 4

From the Departments of Radiological Sciences (Z.P., U.L., A.L., A.S.) and Pathology (D.W.E.), St. Jude Children's Research Hospital, Memphis, Tennessee; and Department of Radiology (J.C.M.), Medical University of South Carolina, Charleston, South Carolina.

Please address correspondence to Zoltan Patay, MD, PhD, Department of Radiological Sciences, St. Jude Children's Research Hospital, 262 Danny Thomas PI, Mail Stop 210, Memphis, TN 38105; e-mail: Zoltan.Patay@STJUDE.ORG

Indicates open access to non-subscribers at www.ajnr.org

http://dx.doi.org/10.3174/ajnr.A2869 literature, in which the diagnosis of the metabolic disorder was established with the necessary degree of confidence. ${ }^{1-40}$ A cerebral neoplasm developed in 14 patients. Seven of the cases were described in case reports; 1 report described a series of 4 patients; and as mentioned above, our 3 patients were taken from a cohort of 11 .

Based on these data, the overall prevalence of brain tumors in patients with L2-HGA would be estimated at $<5 \%$. There is no apparent sex predisposition to the carcinogenic effect.

Analysis of patient age at diagnosis reveals some interesting additional phenomena. Tumors occurred between the ages of 3 and 36 years but were not evenly distributed across this age range; indeed, 3 putative subgroups may be identified within the cohort. Tumors in the youngest patients probably correspond to medulloblastomas (2 patients; the age of 1 of these patients is not known). ${ }^{7}$ Three of the 4 patients with low-grade supratentorial tumors (astrocytoma or lowgrade astrocytoma) were children between 9 and 12 years of age. ${ }^{1,10}$ Only 1 low-grade glioma was found in an adult. Tumors encountered in older teenagers or young adults tended to be high-grade gliomas, though PNETs were also represented. ${ }^{1}$ In 1 patient, neither age at tumor diagnosis nor pathologic diagnosis was available. ${ }^{1}$ Supratentorial tumors occurred in patients older than 9 years, were located in the frontal or temporal lobe or within the thalamus, and were gliomas.

\section{Discussion}

L2-HGA is a rare panethnic organic aciduria. A finding of urinary excretion of 2-hydroxyglutaric acid is required for a formal diagnosis and is accomplished by using chiral gas chromatography and mass spectrometry. All 295 patients reviewed here had the L-isomer variant of 2-hydroxyglutaric aciduria (D-2-hydroxyglutaric aciduria is a very rare inborn error of metabolism, with only 75 cases in the literature). To the best of our knowledge, there have been no reports linking neoplasms of any kind to D-2-hydroxyglutaric aciduria, and this also applies to the extremely rare dual disease, L- and D-2-hydroxyglutaric aciduria. ${ }^{41}$

L2-HGA is a peculiar organic acidopathy in many ways. 

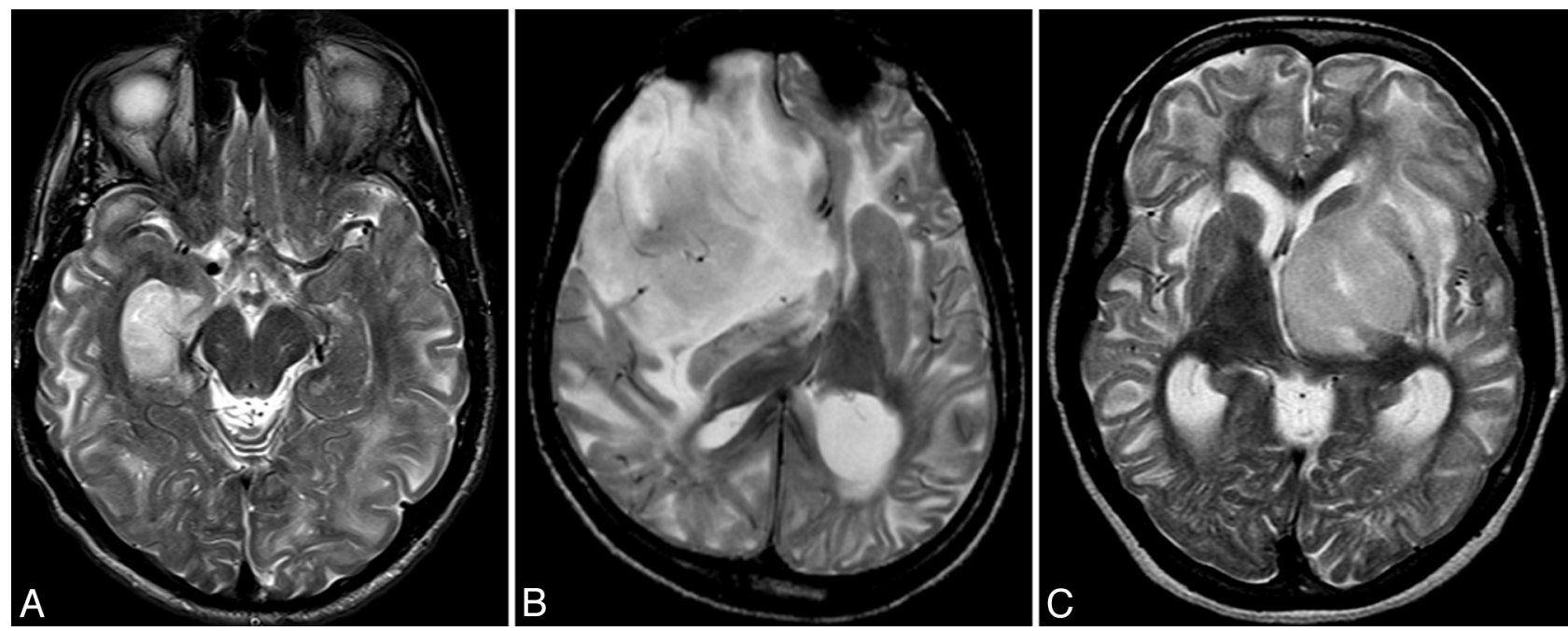

Fig 1. Axial T2-weighted MR images of our patients, Patient $1(A)$ had anaplastic astrocytoma in the right hippocampal area (Fig 2), patient $2(B)$ was diagnosed with oligodendroglioma, and patient $3(C)$ had a low-grade glioma in the left thalamus. Besides the obvious intra-axial mass lesions in all 3 cases, extensive, leukodystrophy-like changes, showing a centripetal gradient are clearly conspicuous; and in conjunction with additional abnormalities found in basal ganglia and dentate nuclei (not shown), these are pathognomonic for L2-HGA.

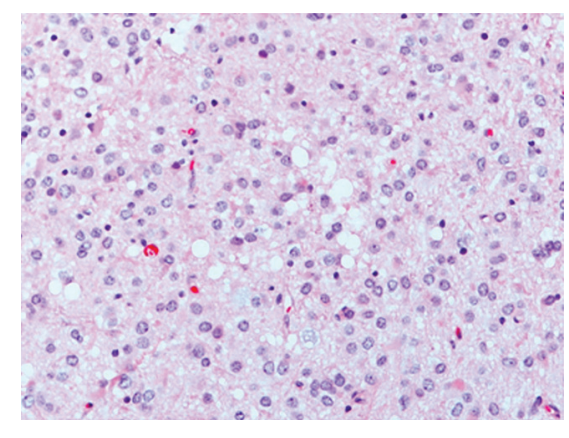

Fig 2. Histopathologic specimen of patient 1 diagnosed with right hippocampal neoplasm. It revealed a diffuse astrocytic tumor characterized by mildly pleomorphic neoplastic cells, microcysts, and some mitotic figures. Hematoxylin-eosin staining, $200 \times$ magnification.

Unlike most other organic acidopathies, it develops insidiously, without episodes of metabolic decompensation. Clinically, it presents with slowly progressive encephalopathy, pyramidal and cerebellar signs, and intellectual deterioration. Thus, patients, though affected to varying degrees neurologically, typically receive a diagnosis late in childhood and often live into adulthood. The disease is associated with the optical isomer of 2-HG (D-2-hydroxyglutaric aciduria) and may have a less benign clinical course.

Abnormalities on MR imaging in L2-HGA are characteristic and, at later stages of the disease, even pathognomonic; hence, the diagnosis is quite often suggested by the radiologist, to be confirmed by laboratory tests. Our 3 cases illustrate this well. Despite the presence of an obvious neoplastic process and its secondary effects (eg, peritumoral vasogenic edema), the background abnormalities of L2-HGA were easy to recognize. Remarkably, a neurometabolic disease was unknown in patient 3 until a left thalamic tumor and L2-HGA were diagnosed, both through the use of MR imaging, when she was 36 years old.

The typical imaging presentation of L2-HGA includes an extensive leukoencephalopathy exhibiting a centripetal and anteroposterior gradient, as well as bilateral symmetric involvement of the globus pallidus, caudate nucleus and putamen, and (characteristically) the cerebellar dentate nuclei. ${ }^{42}$
The leukodystrophy-like appearance of the disease by imaging is unique among organic acidopathies, with the exception of Canavan disease, which also presents with white matter disease and characteristic involvement of the globus pallidus and thalamus (but sparing of the striatum).

From our meta-analysis, an association between L-2-hydroglutaric aciduria and primary cerebral tumors is obvious. Regarding the estimated prevalence of about $5 \%$, it may be either an overestimate or an underestimate. On one hand, it is true that not all L2-HGA cases are necessarily reported, especially in recent years; therefore, the actual prevalence may be lower. However, if one looks at 2 specific comprehensive series (ours and that of Moroni et $\mathrm{al}^{1}$ ), 7 cerebral neoplasms were found among 18 patients, and the actual prevalence may be closer to $40 \%$. It is, of course, possible that an inadvertent accrual bias (ie, patients had more severe disease and hence were more prone to neoplasms) could account for the apparent higher prevalence in these 2 cohorts compared with the rest of the reported cases. Another explanation for the relative under-reporting of an association between L2-HGA and brain tumors is that the metabolic disorder is typically encountered in childhood; therefore, most published cases are likely to have been reported by pediatricians or metabolic specialists. Because the disease is untreatable and is not complicated by episodes of metabolic decompensation later in life, it is possible that discontinuity of care by the time a brain tumor develops in a patient prevents the association being made by the neurosurgeon or radiation oncologist who manages the new disease episode. In this context, it is perhaps relevant that the 2 largest series and the most compelling data come from large referral centers where pediatric neurologists, neurosurgeons, neuropathologists, and neuroradiologists work closely in multidisciplinary teams. In any case, a more systematic follow-up (perhaps including periodic brain MR imaging screening) of patients with L2-HGA may allow us in the future to determine the true prevalence of cerebral neoplasms associated with this metabolic disorder.

Although various theories have been proposed for the association between L2-HGA and brain tumors, the mechanism 


\begin{tabular}{|c|c|c|c|c|c|}
\hline \multicolumn{6}{|c|}{ Clinical and histopathologic data on patients reported in the literature with L2-HGA and cerebral tumors } \\
\hline Authors/Year/Journal & Sex & $\begin{array}{l}\text { L2-HGA, } \\
\text { D2-HGA }\end{array}$ & $\begin{array}{l}\text { Age } \\
\text { (yr) }\end{array}$ & Tumor Histology & Tumor Location \\
\hline$\overline{\text { Aghili et al, 2009, J Neurooncol }{ }^{2}}$ & M & L & 17 & Anaplastic ependymoma & Frontal lobe \\
\hline Wilcken et al, 1993, J Inher Metab Dis ${ }^{32}$ & $\mathrm{~F}$ & L & 17 & PNET & Temporal \\
\hline Barbot et al, 1997, Brain Dev ${ }^{25}$ & $\mathrm{~F}$ & L & 10 & Astrocytoma & Right thalamus \\
\hline Ozişik et al, 2002, Pediatr Neurosurg ${ }^{36}$ & M & L & 3 & Medulloblastoma & Posterior fossa \\
\hline Moroni et al, 2004, Neurology & M & $\mathrm{L}$ & 13.5 & PNET & Right temporal lobe \\
\hline Moroni et al, 2004, Neurology ${ }^{1}$ & $\mathrm{~F}$ & L & 26 & Glioblastoma & Left temporal lobe \\
\hline Moroni et al, 2004, Neurology ${ }^{1}$ & $\mathrm{~F}$ & L & $\mathrm{N} / \mathrm{A}$ & $\mathrm{N} / \mathrm{A}$ & Right temporal lobe \\
\hline Moroni et al, 2004, Neurology' & M & L & 12 & Low-grade astrocytoma & Left frontal lobe \\
\hline Wanders et al, 1997, J Inherit Metab Dis ${ }^{10}$ & $\mathrm{~F}$ & L & 9 & Low-grade astrocytoma & Right thalamus \\
\hline Haliloglu et al, 2008, Neuropediatrics² & M & L & $\mathrm{N} / \mathrm{A}$ & Glioblastoma & Right temporoparietal lobe \\
\hline Topcu et al, 2005, Turk J Pediatr ${ }^{7}$ & $\mathrm{~N} / \mathrm{A}$ & L & $\mathrm{N} / \mathrm{A}$ & Medulloblastoma & N/A \\
\hline Personal series patient 1 & M & L & 23 & Anaplastic astrocytoma & Right hippocampus \\
\hline Personal series patient 2 & M & L & 19 & Oligodendroglioma & Cerebral \\
\hline Personal series patient 3 & $\mathrm{~F}$ & L & 36 & Low-grade glioma & Cerebral \\
\hline
\end{tabular}

Note:-D2-HGA indicates the optic isomer of 2-HG; N/A, not applicable.

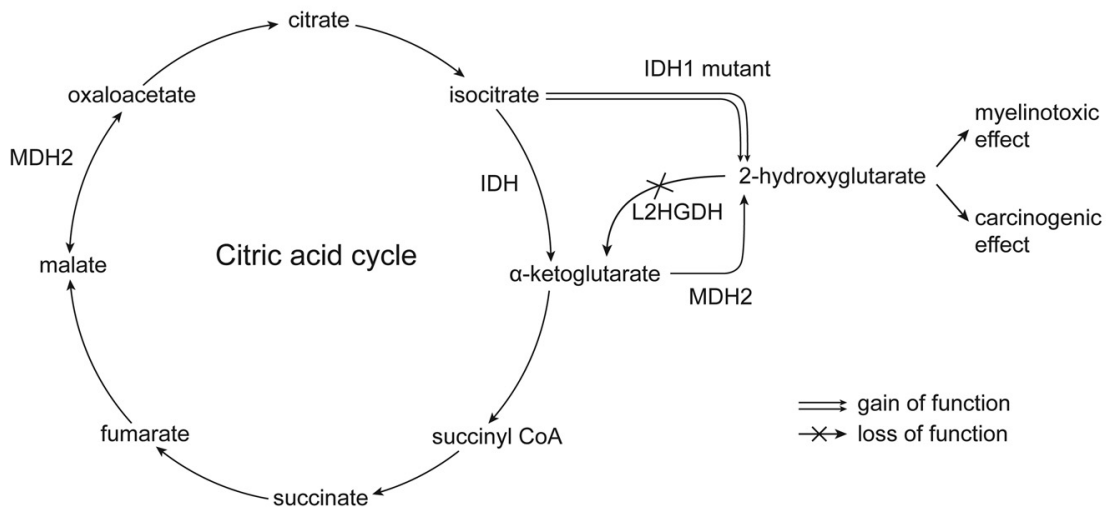

Fig 3. The diagram shows the derivation of 2-HG. With an IDHI mutation and gain of enzyme function, there is an overproduction of 2-HG beyond the capacity of L2HGDH to convert it to alpha-ketoglutarate. In the setting of L2-GA (ie, L2HGDH deficiency), a normal amount of 2-HG is produced, but it accumulates because it is not converted to alpha-ketoglutarate. It is, therefore, excess 2-HG that is both myelinotoxic and carcinogenic. Also shown is the dual function of malate dehydrogenase-2, which converts malate to oxaloacetate as well as alpha-ketoglutarate to $2-\mathrm{HG}$ (there is also a cytosolic form of $\mathrm{MDH}$, which plays a role in gluconeogenesi ${ }^{46}$ ).

of tumorigenesis has only recently been established. Inborn errors of metabolism lead to an accumulation of some metabolites upstream of the defect in the metabolic pathway; in L2HGA, other metabolites in excess but without obvious tumorigenic effect, include glycolate, glycerate, pyruvate, laurate, hydroxybutyrate, citrate, isocitrate, palmitate, myristate, oleate, and stearate. ${ }^{7}$ However, recent studies involving novel sequencing strategies, which were directed first at glioblastoma, uncovered a high frequency of $I D H$ mutations in diffuse gliomas of WHO grade II/III and secondary glioblastomas. More than $80 \%$ of these tumors harbor an IDH1 mutation, which mainly involves 1 codon $(\mathrm{R} 132 \mathrm{H})$ and induces a gain of function ${ }^{43-45}$ that promotes conversion of isocitrate to excessive (nonphysiologic) quantities of potentially carcinogenic 2-HG (IDH normally converts isocitrate to alpha-ketoglutarate in the citric acid cycle).

In addition to its primary function of converting malate to oxaloacetate in the citric acid cycle, ${ }^{46} \mathrm{MDH}$ converts small amounts of alpha-ketoglutarate to 2-HG under normal physiologic conditions. This conversion of alpha-ketoglutarate to 2-HG is insignificant under physiologic conditions because 2-HG is reconverted to alpha-ketoglutarate by $\mathrm{L} 2 \mathrm{HGDH}$ as a metabolic "repair" or "clean-up" mechanism (Fig 3). However, in L2-HGA, 2-HG accumulates and may reach patho- logic and, hence, potentially carcinogenic concentrations because of a mutation in the $L 2 H G D H$ gene at $14 \mathrm{q} 22$ that causes loss of function of L2HGDH. ${ }^{8,47}$ The significance of these physiologic intracellular "housecleaning" processes is being increasingly recognized, and L2-HGA is a prime example of the failure of one such mechanism with subsequent deleterious consequences. In patients with L2-HGA, the mechanism of the 2-HG increase is, therefore, very different from that seen in IDH1 mutants, but the end result is the same.

As described earlier, of the 14 brain tumors encountered in patients with L2-HGA, 9 were diffuse gliomas (WHO grades II, III, IV) of a type found to harbor IDH1 mutations in the sporadic disease. ${ }^{48}$ Medulloblastomas and PNETs seem to occur more frequently in patients with L2-HGA; however, IDH1 mutations have not been reported in medulloblastomas or other PNETs. Finally, although other tumors (bone tumors and Wilms tumor) have also been reported in L2-HGA, these tumors are so rare that these occurrences may be merely coincidental. ${ }^{9,49,50}$

The oncogenetic and myelinotoxic mechanisms induced by accumulation of 2-HG in L2-HGA remain unclear, though the former appears to target glia and the latter has a peculiar lesion pattern with sparing of white matter in the brain stem, cerebellum, corpus callosum, pyramidal tracts, and periven- 
tricular white matter, which constitutes the basis of the diagnosis of the disease by MR imaging. This selective vulnerability also suggests that myelin produced early in life (before the onset of the metabolic derangement and the myelinotoxic effect) may be somewhat resistant to the myelinotoxic pathogenic process but not necessarily to the oncogenetic effect. It is interesting to speculate that in L2-HGA, the short-term effect of excess 2-HG in brain parenchyma may be essentially myelinotoxic in vulnerable areas, whereas long-term exposure of the brain to 2-HG induces neoplastic transformation of glial progenitors or less committed neural stem cells, but to date, no data exist to support this hypothesis.

This review of the clinicopathologic features of patients with L2-HGA who occasionally have brain tumors highlights associations between the metabolic consequences of this disorder and IDH1 mutations in diffuse gliomas, though we must await a clearer understanding of the pathogenesis.

\section{References}

1. Moroni I, Bugiani M, D'Incerti L, et al. L-2-hydroxyglutaric aciduria and brain malignant tumors: a predisposing condition? Neurology 2004;62:1882-84

2. Haliloglu G, Jobard F, Oguz KK, et al. L-2-hydroxyglutaric aciduria and brain tumors in children with mutations in the L2HGDH gene: neuroimaging findings. Neuropediatrics 2008;39:119-22

3. Yazici N, Sarialioglu F, Alkan O, et al. Glutaric aciduria type II [corrected] and brain tumors: a case report and review of the literature. J Pediatr Hematol Oncol 2009;31:865-69. Erratum in J Pediatr Hematol Oncol 2010:32:331

4. Rzem R, Van SE, Veiga-da-Cunha M. The gene mutated in 1-2-hydroxyglutaric aciduria encodes 1-2-hydroxyglutarate dehydrogenase. Biochimie 2006;88: 113-16

5. Rzem R, Vincent MF, Van SE, et al. L-2-hydroxyglutaric aciduria, a defect of metabolite repair. J Inherit Metab Dis 2007;30:681-89

6. Duran M, Kamerling JP, Bakker HD, et al. L-2-hydroxyglutaric aciduria: an inborn error of metabolism? J Inherit Metab Dis 1980;3:109-12

7. Topcu M, Aydin OF, Yalcinkaya C, et al. L-2-hydroxyglutaric aciduria: a report of 29 patients. Turk J Pediatr 2005;47:1-7

8. Topcu M, Jobard F, Halliez S, et al. L-2-hydroxyglutaric aciduria: identification of a mutant gene C14orf160, localized on chromosome 14q22.1. Hum Mol Genet 2004;13:2803-11

9. Rogers RE, Deberardinis RJ, Klesse LJ, et al. Wilms tumor in a child with L-2hydroxyglutaric aciduria. Pediatr Dev Pathol 2010;13:408-11. Epub 2010 Jan 11

10. Wanders RJ, Vilarinho L, Hartung HP, et al. L-2-hydroxyglutaric aciduria: normal L-2-hydroxyglutarate dehydrogenase activity in liver from two new patients. J Inherit Metab Dis 1997;20:725-26

11. Chen E, Nyhan WL, Jakobs C, et al. 1-2-hydroxyglutaric aciduria: neuropathological correlations and first report of severe neurodegenerative disease and neonatal death. J Inherit Metab Dis 1996;19:335-43

12. Aghili M, Zahedi F, Rafiee E. Hydroxyglutaric aciduria and malignant brain tumor: a case report and literature review. J Neurooncol 2009;91:233-36

13. Jequier Gygax M, Roulet-Perez E, Meagher-Villemure K, et al. Sudden unexpected death in an infant with L-2-hydroxyglutaric aciduria. Eur J Pediatr 2009;168:957-62

14. Periasamy V, Rudwan M, Yadav G, et al. Epilepsy in a young adult caused by L-2-hydroxyglutaric aciduria: a case report. Med Princ Pract 2008;17:258-61

15. Sener RN. L-2 hydroxyglutaric aciduria: proton magnetic resonance spectroscopy and diffusion magnetic resonance imaging findings. J Comput Assist Tomogr 2003;27:38-43

16. Karatas H, Saygi S, Bastan B, et al. L-2-hydroxyglutaric aciduria: report of four Turkish adult patients. Neurologist 2010;16:44-46

17. Saidha S, Murphy S, McCarthy P, et al. L-2-hydroxyglutaric aciduria diagnosed in an adult presenting with acute deterioration. J Neurol 2010;257:146-48

18. Clerc C, Bataillard M, Richard P, et al. An adult form of L-2-hydroxyglutaric aciduria revealed by tremor. Eur Neurol 2000;43:119-20

19. Mahfoud A, Dominguez CL, Perez A, et al. L-2-hydroxyglutaric aciduria: clinical, biochemical and neuroradiological findings in two Venezuelan patients [in Spanish]. Rev Neurol 2004;39:343-46

20. Larnaout A, Hentati F, Belal S, et al. Clinical and pathological study of three Tunisian siblings with L-2-hydroxyglutaric aciduria. Acta Neuropathol 1994;88:367-70
21. Barth PG, Hoffmann GF, Jaeken J, et al. L-2-hydroxyglutaric acidaemia: clinical and biochemical findings in 12 patients and preliminary report on L-2hydroxyacid dehydrogenase. J Inherit Metab Dis 1993;16:753-61

22. Barth PG, Hoffmann GF, Jaeken J, et al. L-2-hydroxyglutaric acidemia: a novel inherited neurometabolic disease. Ann Neurol 1992;32:66-71

23. Sztriha L, Gururaj A, Vreken P, et al. L-2-hydroxyglutaric aciduria in two siblings. Pediatr Neurol 2002;27:141-44

24. Zafeiriou DI, Sewell A, Savvopoulou-Augoustidou P, et al. L-2-hydroxyglutaric aciduria presenting as status epilepticus. Brain Dev 2001;23:255-57

25. Barbot C, Fineza I, Diogo L, et al. L-2-hydroxyglutaric aciduria: clinical, biochemical and magnetic resonance imaging in six Portuguese pediatric patients. Brain Dev 1997;19:268-73

26. D’Incerti L, Farina L, Moroni I, et al. L-2-hydroxyglutaric aciduria: MRI in seven cases. Neuroradiology 1998;40:727-33

27. Seijo-Martinez M, Navarro C, Castro del RM, et al. L-2-hydroxyglutaric aciduria: clinical, neuroimaging, and neuropathological findings. Arch Neurol 2005;62:666-70

28. Shafeghati Y, Vakili G, Entezari A. L-2-hydroxyglutaric aciduria: a report of six cases and review of the literature. Arch Iran Med 2006;9:165-69

29. Goffette SM, Duprez TP, Nassogne MC, et al. L-2-hydroxyglutaric aciduria: clinical, genetic, and brain MRI characteristics in two adult sisters. Eur J Neurol 2006;13:499-504

30. Haworth JC, Dilling LA, Seargeant LE. Increased prevalence of hereditary metabolic diseases among native Indians in Manitoba and Northwestern Ontario. CMAJ 1991;145:123-29

31. Rzem R, Veiga-da-Cunha M, Noel G, et al. A gene encoding a putative FADdependent L-2-hydroxyglutarate dehydrogenase is mutated in L-2-hydroxyglutaric aciduria. Proc Natl Acad Sci U S A 2004;101:16849-54. Epub 2004 Nov 17

32. Wilcken B, Pitt J, Heath D, et al. L-2-hydroxyglutaric aciduria: three Australian cases. J Inherit Metab Dis 1993;16:501-04

33. Lee C, Born M, Salomons GS, et al. Hemiconvulsion-hemiplegia-epilepsy syndrome as a presenting feature of L-2-hydroxyglutaric aciduria. J Child Neurol 2006;21:538-40

34. Fujitake J, Ishikawa Y, Fujii H, et al. L-2-hydroxyglutaric aciduria: two Japanese adult cases in one family. J Neurol 1999;246:378-82

35. Warmuth-Metz M, Becker G, Bendszus M, et al. Spinal canal stenosis in L-2hydroxyglutaric aciduria. Arch Neurol 2000;57:1635-37

36. Ozişik PA, Akalan N, Palaoğlu S, et al. Medulloblastoma in a child with the metabolic disease L-2-hydroxyglutaric aciduria. Pediatr Neurosurg 2002; 37:22-26

37. de Klerk JB, Huijmans JG, Stroink H, et al. L-2-hydroxyglutaric aciduria: clinical heterogeneity versus biochemical homogeneity in a sibship. Neuropediatrics 1997;28:314-17

38. Topcu M, Erdem G, Saatci I, et al. Clinical and magnetic resonance imaging features of L-2-hydroxyglutaric acidemia: report of three cases in comparison with Canavan disease. J Child Neurol 1996;11:373-77

39. Hanefeld F, Kruse B, Bruhn $\mathrm{H}$, et al. In vivo proton magnetic resonance spectroscopy of the brain in a patient with L-2-hydroxyglutaric acidemia. Pediatr Res 1994;35:614-16

40. Moroni I, D'Incerti L, Farina L, et al. Clinical, biochemical and neuroradiological findings in L-2-hydroxyglutaric aciduria. Neurol Sci 2000;21:103-08

41. Muntau AC, Roschinger W, Merkenschlager A, et al. Combined D-2- and L-2hydroxyglutaric aciduria with neonatal onset encephalopathy: a third biochemical variant of 2-hydroxyglutaric aciduria? Neuropediatrics 2000; 31:137-40

42. van der Knaap MS. L-2-hydroxyglutaric aciduria. In: van der Knaap MS, Valk J, eds. Magnetic Resonance of Myelination and Myelin Disorders. 3rd ed. Berlin, Germany: Springer-Verlag; 2005:334-37

43. Dang L, White DW, Gross S, et al. Cancer-associated IDH1 mutations produce 2-hydroxyglutarate. Nature 2009;462:739-44

44. Parsons DW, Jones S, Zhang X, et al. An integrated genomic analysis of human glioblastoma multiforme. Science 2008;321:1807-12

45. Balss J, Meyer J, Mueller W, et al. Analysis of the IDH1: codon 132 mutation in brain tumors. Acta Neuropathol 2008;116:597-602. Epub 2008 Nov 5

46. Musrati RA, Kollarova M, Mernik N, et al. Malate dehydrogenase: distribution, function and properties. Gen Physiol Biophys 1998;17:193-210

47. Van SE, Rzem R, Veiga-da-Cunha M. L-2-Hydroxyglutaric aciduria, a disorder of metabolite repair. J Inherit Metab Dis 2009;32:135-42

48. Yan H, Parsons DW, Jin G, et al. IDH1 and IDH2 mutations in gliomas. NEngl J Med 2009;360:765-73

49. Gross S, Cairns RA, Minden MD, et al. Cancer-associated metabolite 2-hydroxyglutarate accumulates in acute myelogenous leukemia with isocitrate dehydrogenase 1 and 2 mutations. J Exp Med 2010;207:339-44

50. Larnaout A, Amouri R, Neji S, et al. Osteoma of the calvaria in L-2-hydroxyglutaric aciduria. J Inherit Metab Dis 2007;30:980 